\title{
Pris au dépourvu par l'arrivée de maladies tropicales
}

\author{
Niranjan Kissoon, MBBS*; Tim Uyeki, $\mathrm{MD}^{\dagger}$
}

La déréglementation du transport aérien, entrée en vigueur en 1978, et l'optimisation des voies aériennes ont eu un effet des plus avantageux sur les voyages à l'étranger, y compris sur ceux en provenance ou à destination du Canada, pays du G8 qui connaît l'une des plus fortes croissances de la population. ${ }^{1}$ Des millions de touristes provenant de pays outre-mer visitent le Canada chaque année. Par ailleurs, plus de $20 \%$ de la population canadienne (presque 7 millions de personnes) est née à l'étranger, et au moins 50\% des nouveaux immigrants arrivent de régions tropicales. De plus, les immigrants récents peuvent retourner dans leur pays d'origine et en revenir., ${ }^{2,3}$

Il est possible que des voyageurs visitent des pays en voie de développement, dans des régions tropicales ou subtropicales, et soient ainsi exposés à des maladies infectieuses, non endémiques au Canada (ex.: dengue, paludisme, fièvre Chikungunya, fièvre typhoïde, encéphalite japonaise, choléra, rougeole) ou à d'autres maladies peu fréquentes (tuberculose pharmacosensible, multirésistante, ou ultrarésistante). Ainsi, les voyageurs et les migrants atteints de certaines de ces maladies présentent un risque de transmission aux personnes qui entrent en contact étroit avec eux et aux membres du personnel soignant. ${ }^{4}$ L'importation de maladies infectieuses au Canada peut se faire par la voie des airs ou des mers, par l'intermédiaire de passagers qui reviennent de régions du monde où peuvent circuler des virus de maladies respiratoires (ex.: virus de la grippe saisonnière de type A ou B), ou encore par des cliniciens peu méfiants, qui peuvent ne pas administrer le traitement approprié. $^{5,6}$ On peut supposer que la médecine d'urgence au Canada se pratique dans un climat potentiellement souillé de maladies tropicales importées.

Le rapport de Meshkat et de ses collègues, inclus dans le présent numéro de la revue, soutient cette prétention. ${ }^{7}$ Ils ont procédé à une recherche documentaire, ont mené une enquête anonyme en ligne parmi les urgentologues et ont passé en revue les principaux congrès organisés en médecine d'urgence universitaire et les grandes revues médicales afin de cerner les lacunes en matière de connaissances sur des maladies tropicales fréquentes et en matière de traitement. Résultat: ils ont recensé un petit nombre de rapports, qui consistaient presque tous en des exposés de cas rétrospectifs, et un nombre encore plus petit de documents portant sur la médecine d'urgence. Dans l'ensemble, $70 \%$ des répondants ont fait état d'une très faible exposition à ce type de maladies durant leur formation et d'un manque d'assurance dans la prise en charge des maladies tropicales, et la plupart (77\%) estimaient qu'ils avaient besoin de parfaire leurs connaissances en la matière. Peu (6 cours sur 2038) de séances de formation médicale continue (FMC) portaient sur les maladies tropicales à l'intention des médecins d'urgence au Canada. Bien qu'il ne faille pas s'étonner des résultats, ils sont quelque peu embarrassants et sont perçus comme une mauvaise réponse aux scénarios soumis. Malgré le fait que l'étude comporte des faiblesses, un examen approfondi n'aurait pas changé les résultats selon lesquels les urgentologues ne sont pas prêts à traiter des maladies tropicales.

\footnotetext{
*Department of Pediatrics and Emergency Medicine, Faculty of Medicine, University of British Columbia, Vancouver, BC, et + National Center for Immunization and Respiratory Diseases, Centers for Disease Control and Prevention - Influenza Division, Atlanta, GA.
}

Correspondance à: Dr. Niranjan Kissoon, Department of Pediatrics and Emergency Medicine, Faculty of Medicine, University of British Columbia, 4480 Oak Street, Room B 245, Vancouver, BC V6H 3V4.

Cet article a été évalué par des pairs. 
Les résultats de l'étude donnent à penser que certains médecins d'urgence, lorsqu'ils examinent des patients fiévreux, qui souffrent d'une maladie inconnue, contractée dans des régions tropicales, poseraient les mauvaises questions, envisageraient le mauvais diagnostic, ou prescriraient le mauvais traitement, ce qui se traduit par de piètres résultats cliniques (ex.: complications du paludisme non traité, qui mènent à l'hospitalisation dans un service de soins intensifs ou à la mort). Certes, il peut s'agir d'une maladie fréquente dans les pays tropicaux et subtropicaux, mais les médecins au Canada peuvent ne pas bien connaître cette maladie ni son traitement approprié. Pourtant, un mauvais diagnostic d'une maladie susceptible de se transmettre par voie aérienne (ex.: rougeole, tuberculose) risque d'affecter d'autres malades exposés et des membres du personnel soignant qui n'ont pas fait usage du bon matériel protecteur, en plus de mettre en danger les membres de la famille et l'ensemble de la collectivité.

Deux nouveaux virus de maladies respiratoires ont fait leur apparition depuis peu, et ils causent, chez l'homme, des infections graves, même mortelles, des voies respiratoires inférieures. Ainsi, la grippe aviaire de type A (H7N9) chez les hommes (taux de mortalité parmi les cas: $28 \%$ ) serait essentiellement une maladie zoonotique, due à l'exposition à de la volaille infectée en Chine. ${ }^{8-10}$ Jusqu'à maintenant, un cas a été exporté à Taiwan. ${ }^{11}$ Par ailleurs, des cas d'infection par le coronavirus du syndrome respiratoire du MoyenOrient (MERS-CoV) ont été observés au MoyenOrient (Arabie saoudite, Jordanie, Qatar, Émirats arabes unis), puis exportés dans d'autres pays, notamment au Royaume-Uni, en Allemagne, en France, en Italie, et en Tunisie. ${ }^{12-14}$ Le MERS-CoV est génétiquement lié au coronavirus du syndrome respiratoire aigu grave (SRAG), qui rappelle l'éclosion de cette maladie à Toronto, en 2003, mais le taux de mortalité parmi les cas de MERS (46\%), en date du 30 août 2013, est de beaucoup supérieur à celui du SRAG. ${ }^{15}$ On ne connaît pas actuellement la source du MERS-CoV. Des cas isolés de transmission d'homme à homme du virus H7N9 ainsi que du MERS-CoV ont déjà été observés (y compris des cas de transmission nosocomiale), ce qui n'est pas sans soulever des préoccupations en matière de santé publique à l'échelle mondiale., ${ }^{9,13}$ En ce qui concerne ces deux infections virales, une anamnèse qui fait état d'un voyage récent (en Chine pour le virus H7N9 et au Moyen-Orient pour le MERS-CoV), de contact avec les animaux (y compris avec de la volaille), ou d'un contact étroit avec une personne malade est essentielle pour soulever des doutes cliniques. Nombreux sont les Canadiens qui sont allés en Arabie saoudite, de septembre à novembre 2013, pour le grand pèlerinage (Hajj) tenu en octobre, et qui en sont revenus, ce qui soulève des préoccupations quant à la transmission et à l'exportation du MERS-CoV, ainsi qu'à l'extension de son territoire. De pareils cas d'exportation de maladies se sont faits très rares jusqu'à maintenant dans le monde, mais les médecins d'urgence devraient être conscients du risque lorsque des patients arrivent d'autres pays et présentent des maladies respiratoires aiguës fébriles, susceptibles d'être causées par de nouveaux virus s'attaquant aux voies respiratoires.

Il est crucial de respecter les mesures de lutte contre les infections, recommandées dans la prévention de la transmission nosocomiale d'agents pathogènes respiratoires, tant nouveaux que connus, qui sont source de préoccupation en santé publique au service des urgences. ${ }^{16}$ L'application continue, et sans relâche, de mesures normales de précaution, l'isolement ou le regroupement des malades, et la mise en pratique de mesures de précaution relatives au contact avec des patients qui présentent des symptômes de maladie respiratoire et à la contamination par gouttelettes aideront à diminuer le risque de transmission de maladies respiratoires, nouvelles, ou connues. Une surveillance accrue des maladies, fondée sur les laboratoires, et l'établissement de liens étroits entre la collectivité en santé publique et les cliniciens d'urgence s'imposent afin de faciliter une prise en charge clinique appropriée (y compris la lutte contre les infections) et une réaction rapide aux nouvelles menaces qui planent à l'échelle mondiale.

Pour combler les lacunes en matière de prise en charge des infections tropicales, il faudrait envisager une approche à facettes, prévoyant notamment l'adoption de politiques qui réclament une sensibilité accrue à l'égard de la santé mondiale, de la formation en matière de prise en charge des maladies tropicales et de nouvelles maladies infectieuses, et l'enseignement de compétences en matière de prise en charge dans les programmes des écoles de médecine; des programmes de formation au niveau de la résidence; ainsi que la délivrance et le renouvellement du certificat de spécialiste en médecine d'urgence. D'après les résultats de l'étude menée par Meshkat et ses collègues, ${ }^{7}$ la FMC à l'intention des praticiens devrait consister en des exposés de cas, en des séances de formation de type magistral sur certaines maladies tropicales, et en la présentation de différentes conduites à adopter pour la 
prise en charge d'un large éventail de syndromes cliniques. Les séances de FMC devraient reposer non pas sur une seule formule d'enseignement, mais sur plusieurs, telles que les webinaires et la baladodiffusion, moyens accessibles en ligne, ainsi que sur des ateliers organisés dans le cadre de congrès, portant sur la médecine des voyages et préparés en collaboration avec des sociétés médicales établies au Canada ou ailleurs dans le monde. De plus, les comités de rédaction de revues en médecine d'urgence devraient mettre en place une chronique, accessible en ligne et portant sur le diagnostic et le traitement des maladies infectieuses tropicales, et prévoir des mises à jour périodiques sur l'apparition ou la réapparition de nouvelles maladies infectieuses dans le monde. Ce travail pourrait se faire en collaboration avec des infectiologues spécialisés dans la médecine des voyages, des sociétés médicales professionnelles, des services locaux, et provinciaux de santé publique et l'Agence de la santé publique du Canada.

La formation doit inclure la manière de recueillir une anamnèse complète sur les antécédents de voyage. D'après une anamnèse faisant état d'un voyage récent et de manifestations cliniques, notamment d'une maladie fébrile, il faudrait inciter les urgentologues à consulter des spécialistes en médecine des voyages et en infectiologie pour en savoir davantage sur la prise en charge clinique de cas de maladies vraisemblablement liées à des voyages. Un moyen d'augmenter le nombre de questions sur les voyages récents serait d'en faire une exigence pour l'agrément des hôpitaux, dans tous les cas de maladie fébrile, non liée à des traumas, au service des urgences, au moment du triage.

Aucune formule ne fera de nous des spécialistes en maladies tropicales, mais un effort concerté, comprenant différentes formules d'apprentissage est le meilleur moyen de combler les lacunes en matière de connaissances. Les auteurs du rapport tirent la sonnette d'alarme, et il faut améliorer rapidement la formation afin que les professionnels concernés soient en mesure de reconnaître et de traiter les maladies importées, et de les prendre correctement en charge, si rares soient-elles pour la plupart des médecins au Canada. Il ne faudrait pas être pris au dépourvu par l'arrivée inopinée d'une maladie tropicale ou d'un nouveau virus au service des urgences.

Intérêts concurrentiels: Aucun déclaré.

\section{RÉFÉRENCES}

1. Smith FL Jr, Cox B. Airline deregulation. Available at: http:// www.econlib.org/library/Enc/AirlineDeregulation.html (accessed June 18, 2013).
2. Radia A. Canada's population reaches 35 million, fastest growing in the G8. Canada Politics. December 7, 2012. Available at: http://ca.news.yahoo.com/blogs/canada-politics/ canada-population-reaches-35-million-fastest-growing-g8174256519.html) (accessed June 18, 2013).

3. The Canadian Press. Canada's foreign-born population soars to 6.8 million. Available at: http://www.cbc.ca/news/canada/ story/2013/05/08/census-statistics-canada-household-survey. html (accessed June 18, 2013).

4. McCarthy AE, Weld LH, Barnett ED, et al. Spectrum of illness in international migrants seen at GeoSentinel clinics in 19972009, part 2: migrants resettled internationally and evaluated for specific health concerns. Clin Infect Dis 2013;56:925-33.

5. Uyeki TM, Zane SB, Bodnar UR, et al. Alaska/Yukon Territory Respiratory Outbreak Investigation Team. Large summertime influenza A outbreak among tourists in Alaska and the Yukon Territory. Clin Infect Dis 2003;36:1095-102.

6. Belderok SM, Rimmelzwaan GF, van den Hoek A, Sonder GJ. Effect of travel on influenza epidemiology. Emerg Infect Dis 2013;19:925-31.

7. Meshkat N, Misra S, Hunchak C, et al. Knowledge gaps in the diagnosis and management of patients with tropical diseases presenting to Canadian emergency departments: are the gaps being met? Can 7 Emer Med 2014;16:458-66.

8. Gao HN, Lu HZ, Cao B, et al. Clinical findings in 111 cases of influenza A (H7N9) virus infection. N Engl 7 Med 2013 May 22. [Epub ahead of print]

9. Li Q, Zhou L, Zhou M, et al. Preliminary report: epidemiology of the avian influenza A (H7N9) outbreak in China. N Engl 7 Med 2013 Apr 24. [Epub ahead of print]

10. World Health Organization. WHO risk assessment. Human infections with avian influenza A (H7N9) virus 7 June 2013. Available at: http://www.who.int/influenza/human_animal_ interface/influenza_h7n9/RiskAssessment_H7N9_07Jun13. pdf (accessed June 18, 2013).

11. Chang SY, Lin PH, Tsai JC, et al. The first case of H7N9 influenza in Taiwan. Lancet 2013;381:1621.

12. Memish ZA, Zumla AI, Al-Hakeem RF, et al. Family cluster of Middle East respiratory syndrome coronavirus infections. N Engl 7 Med 2013 May 29. [Epub ahead of print]

13. Assiri A, Al-TawfiqJA, Al-Rabeeah AA, et al. Epidemiological, demographic, and clinical characteristics of 47 cases of Middle East respiratory syndrome coronavirus disease from Saudi Arabia: a descriptive study. Lancet Infect Dis 2013;13:752-61.

14. World Health Organization. Middle East respiratory syndrome coronavirus (MERS-CoV) summary and literature update - as of 13 August 2013. Available at: http://www.who.int/csr/ disease/coronavirus_infections/update_20130813/en/index. html (accessed August 30, 2013).

15. World Health Organization. Middle East respiratory syndrome coronavirus (MERS-CoV) - update. Available at: http://www.who.int/ csr/don/2013_08_30/en/index.html (accessed August 30, 2013).

16. World Health Organization. Interim Guidance Document. Clinical management of severe acute respiratory infections when novel coronavirus is suspected: what to do and what not to do. Available at: http://www.who.int/csr/disease/coronavirus_infections/ InterimGuidance_ClinicalManagement_NovelCoronavirus_ 11Feb13u.pdf (accessed June 18, 2013). 